\title{
An Application on Women Safety Using Embedded Systems andIoT
}

\author{
K Hari Kishore ${ }^{1}$, EswarTeja Ravuri ${ }^{2}$, Pavan Kumar Sankarasetty ${ }^{3}$, Vamsi Krishna Mogilicharla ${ }^{4}$, \\ E Raghveera ${ }^{5}$
}

\author{
1,2,3,4,5 Department of ECE, KoneruLakshmaiah Education Foundation,Vaddeswaram, Guntur, \\ AndhraPradesh,India \\ 2ravurieswarteja@gmail.com
}

Article History:Received: 10 November 2020; Revised: 12 January 2021; Accepted: 27 January 2021; Published online: 05 April 2021

\begin{abstract}
As far as women safety is concerned. Especially in our country the standards are going on decreasing day by day. According to National Crime Records Bureau (NCRB) the incidents of kidnapping and abductions rose to 64 per million to 74 per million which is devastating. If it goes on increasing like this, In the coming years there will be a tremendous rise in crime rate especially against women. It is our responsibility to protect our mothers, sisters, friends and each and every woman in our family. There are many devices in the market for this application that is sending locations and alert messages to the family of the user. But, all the devices need the response from the user when they are in danger to send the alert messages or such to their family or emergency services. But there might be situations where the user has zero chances to respond or send any alert messages to their family. We came up with a solution where the alert messages and current location of the user are sent automatically to their family when they are in danger without any response from the user. We achieved it using the pulse rate sensor PPG (Photoplethysmography sensor) using which we measured the abnormal rise in heart rate when the user is in danger. This paper deals with the functionality and implementation of our idea in Embedded Systems and IoT.
\end{abstract}

Keywords:Danger, Kidnapping, Alert Messages, Photoplethysmography Sensors, Embedded Systems, IoT.

\section{Introduction}

Safety standards especially for the women are getting worse day by day. A wide variety of safety devices in different forms with different functionalities are available in the market. These safety devices are available in the form of wearable, mobile applications,key chains, lipsticks, torch lights and many. But, all these devices require user's interaction in the time of emergency that is the user should press the button present in the device twice or thrice or send messages through any mobile application or ringing any buzzers that too by pressing some buttons. But, every situationis not that favorable for the user to respond immediately. Or there might not be a chance for the user to press the buttons present on the device or take the mobile phone out to send the alert messages. There are situations where the kidnappers can tie the user's hands off. Which restricts the users from interacting with safety device present with them? Users cannot completely rely on such devices which require the user's interaction.There is a requirement of a device which works effectively and sends the alert messages even without any user's interaction. Then after a long research we came up with an idea to design a safety device using PPGS (Photoplethysmography sensors) which is used for pulse rate monitoring in different medical devices, fitness trackers, blood oxygen level monitors. Our idea was to design the device which relies on the heart rate of the user.The first thing that happens when a person encounters a danger is that the pulse rate starts running marathon. It will go abnormal for the amount of time until the user gets normal. The heart rate remains high as long as the user is in the tensed state. This is what the base for our project is. Measuring the heart rate of the person using PPGS if it is high for more than ample amount of time and there isn't any interaction from the user we will send the alert message along with the location to the family members and emergency services.For the time being we have designed the device for the women below 30 years without any health complications. Using these PPGS for fingers. Where, we can get the pulse rate of a person. We are trying to develop this in the form of a wearable which can be worn on hand and which even can't be identified as a tracking or safety device.

\section{Literature Review}

Many of the top most watch companies have designed devices for this application. They have added a button to their watches and provided them with Bluetooth connectivity so that the watch sends the alert messages by the means of connected Bluetooth and mobile phone. In these particular models also the user has to press a button present on the watch. And many mobile phone companies also included a feature in their mobiles. Like, if the user is in danger the user should press the power button on the mobile for three times. These may not work in everyone situation.In[1] they have placed an emergency button in their setup if the user is in danger then the user should press the button present in the setup. Upon successful pressing of that button for 2-3 times it sends the alert messages to the emergency contacts.

In [2] they have designed the device in the form of a ring this also needs the physical attention of the user. There is a button present in the ring; on pressing the button on the ring alert messages are sent to the emergency 
contacts. In [3] they also implanted a button in the device on pressing that button emergency messages are sent. In [4] they have designed the safety device in the form of a footwear which also requires the physical attention of the user. In[4] the user should apply pressure on the footwear or press the button implanted in it. In [5] they have also implemented the model which functions only on the user's interaction. Upon interaction by means of a button by the user it sends alert messages. Almost all the existing devices which are currently available in the market work on this very principle. In [6] they have designed a mobile application which requires user's interaction. The user must press the button present in the application which sends alert messages.[7] grabbed our attention they have designed a device which sends alert messages automatically based on the heart rate of the user. They set a target heart rate and if that target is achieved for some time then they sent alert messages directly to the family. But, pulse rate rises not only at the time of fear there are many possibilities for the pulse rate to rise. That might be during physical workout or when giving a speech on an unknown topic in an auditorium infront the crowd without any prior preparation. So there is a requirement of an intelligent device which should be designed in such a way that the user can snooze the device for required amount of time if the user know in prior that the user is going to attend such events where there are chances of the rise in pulse rate and even in the sleep state the device should protect the user and also the device must be user friendly and work in maximum situations possible.

\section{Proposed Design}

In this paper we have proposed a methodology which works in almost all situations possible. For time being we have designed this prototype for age group below 30 without any health complications. Figure 1 gives the details of the implementation of the setup which we have designed.

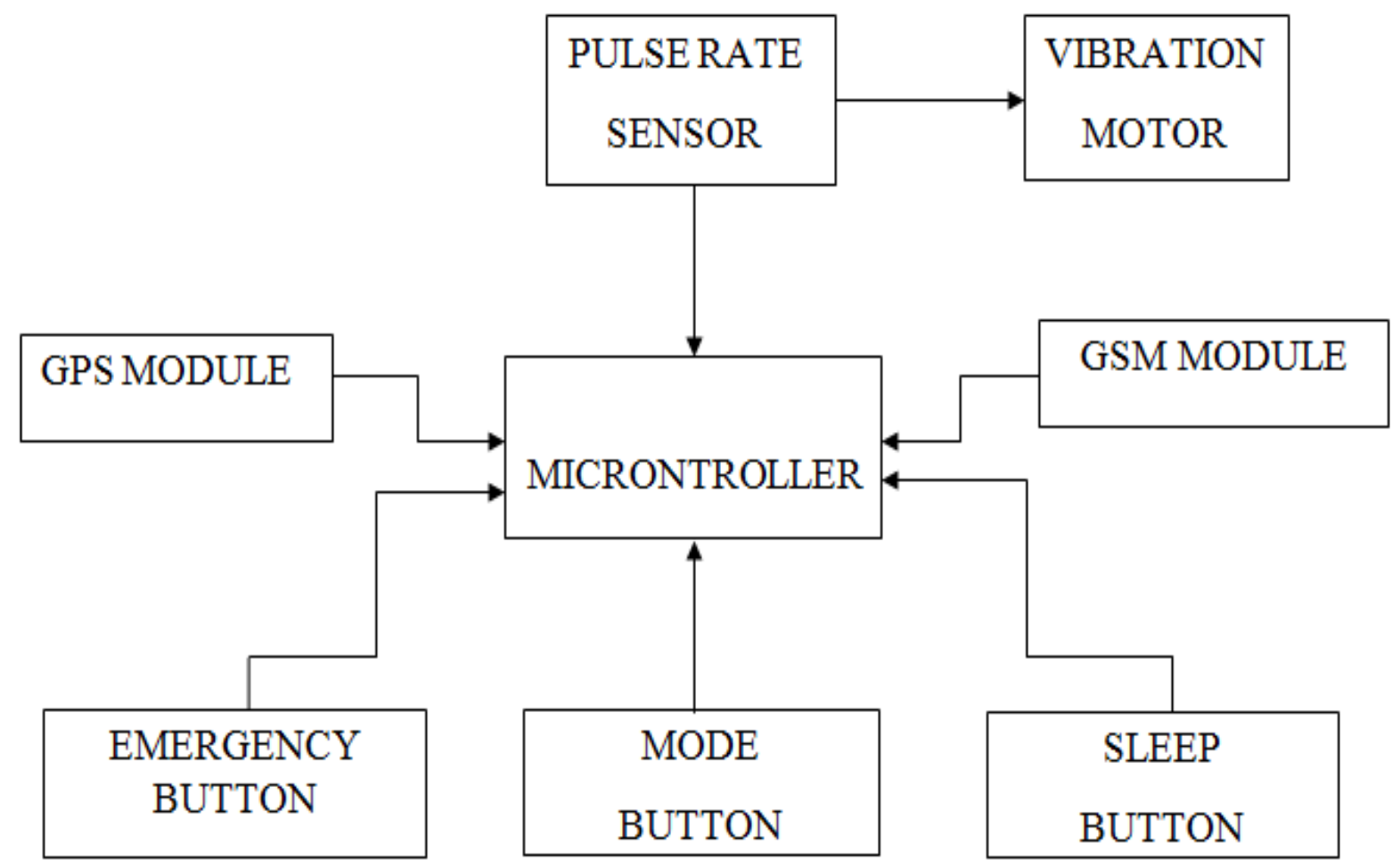

Figure 1. Block Diagramofthe Proposed Design

This unit basically consists of a microcontroller which is the heart of any project. It consists of a pulse rate sensor PPG (Photoplethysmography sensor) used for the application of measuring pulse rate in fitness bands and medical grade pulseoxymeters. It comprises of a network of GPS and GSM modules for sending alert messages and location of the user. It consists of a vibration motor and three pushbuttons which are of different functionality and the functionality of the buttons is explained below and the working methodology of the proposed design is shown in the Figure 2. 


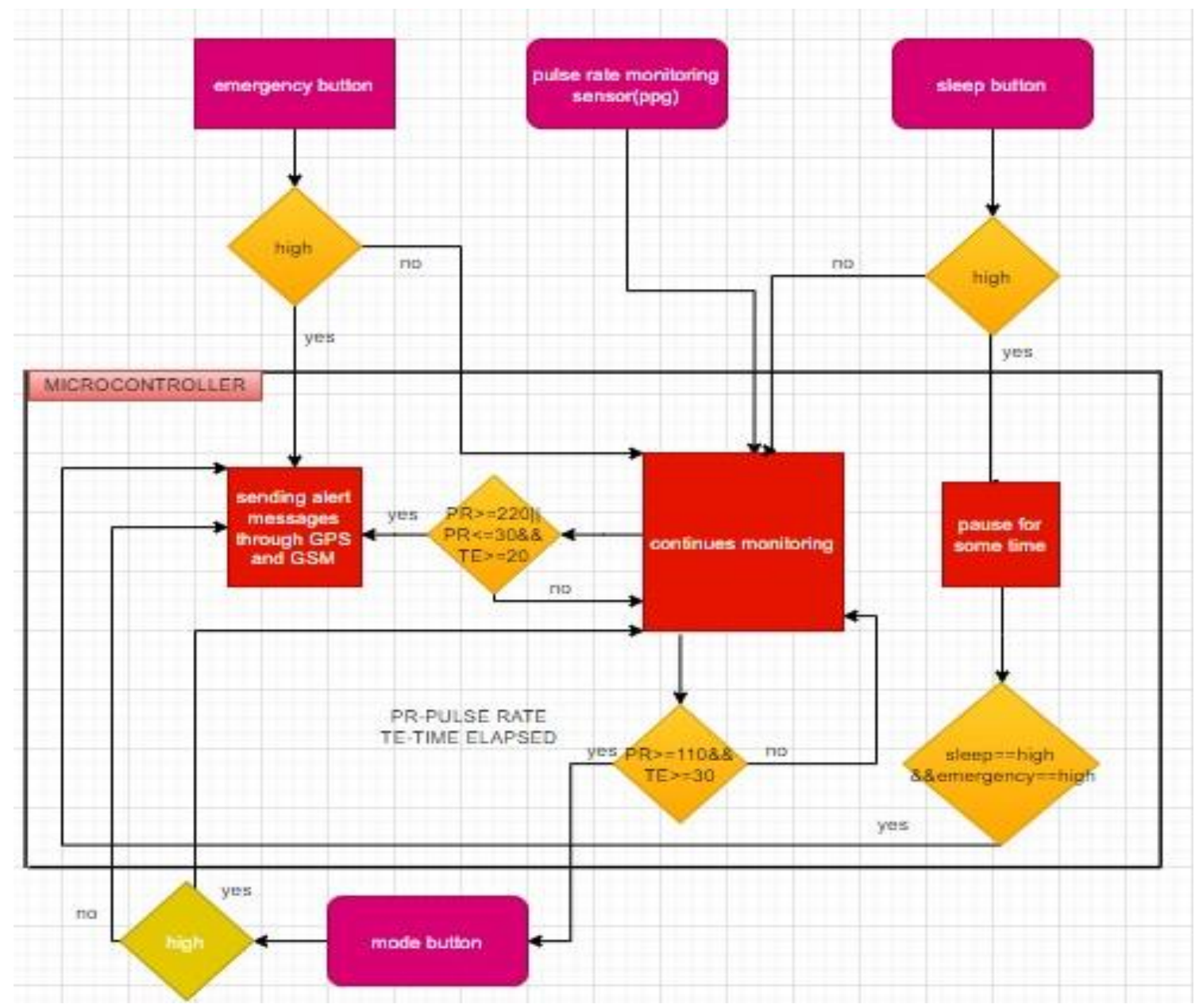

Figure 2.Flow Chart of Methodology

This device basically works on the principle that during the dangerous situation like kidnapping or sexual harassment the fight or flight response activates as a result the pulse rate of the user goes high. This will not at all come to normal state until the user comes back into normal state that is out of fear. We have set a value of 120 as the threshold heart rate. If that particular heart rate is achieved the device checks whether the users heart rate is above the threshold heart rate value. If it is the case, the device will check for the next thirty seconds whether the heart rate of the user Is above the threshold heart rate value. If the user's heart rate value is above 120 for 20 seconds at least. The device goes into the second stage of working. In the second stage for the next thirty seconds the device starts vibrating with the help of vibration motor once in every 2 seconds this vibration is felt on the hand only by the user. The second stage is for user's conformation in order to eradicate the false triggers. That is if the user is in a position to respond then, the user will click the mode button in the setup. Just after clicking the mode button the watch resets and it starts monitoring the heart rate right from the beginning. If the user did not respond even after the 30 seconds of continuous vibration then the device confirms that the user is in danger and it automatically sends the alert message along with the GPS location of the user using the GPS and GSM module.

\subsection{Functionality of the Hardware present in the device}

A) Mode Button: As we mentioned above, when the heart rate of the person goes beyond the abnormal heart rate value which is 110 in our case and if it lasts more than 110 for at least 30 seconds. The vibration motor starts vibrating for every 2 seconds for the next 30 seconds. If the user is in a state to respond then the user responds through this Mode Button. If the user presses the button once after the vibration motor starts then the device gets reset automatically and it starts taking the fresh readings once again. If the user is not in a position to press the button then it automatically sends the alert messages to the emergency contacts. Upon using this button we can eradicate false triggers by getting the user's confirmation which may sometimes be fatal. 
B) Sleep Button: This button provides us with the sleep functionality. Pulse rate of a person not only rises when we face emotions like fear or anger it rises during physical workouts also. There are some situations where our pulse rate rises for instance working out in a gym rise our pulse rate due to consumption of excess oxygen required for the body. And situations which make us nervous like presenting something unknown in front of a crowd in an auditorium. In such situations where we know that our heart rate rises in prior. On pressing this button it asks the user to enter the time for which the user wants the device not to monitor his/her pulse rate. After the completion of the entered time the device automatically does its duty of monitoring the pulse rate of the user.

Note:during the sleep stage of the device the emergency button functions. If the user is in danger when the device is in sleep mode the user just has to press the emergency button for the help.

C) Emergency Button: This button will be available for the user once after the board is powered. If the user is in danger and if he/she has time to respond immediately then the user should press this button in order to get the instant help form the emergency contacts. Upon long pressing this button the microcontroller sends the alert messages and location directly to the emergency contacts.

D) Pulse Rate Sensor: These are Photoplethysmography sensors which are widely used for measuring pulse rate in humans especially in the form of wearable and medical grade pulseoximeters. These are used in our device to measure the pulse rate of the user continuously. Pulse rate sensor used in our device is shown in the Figure 3.

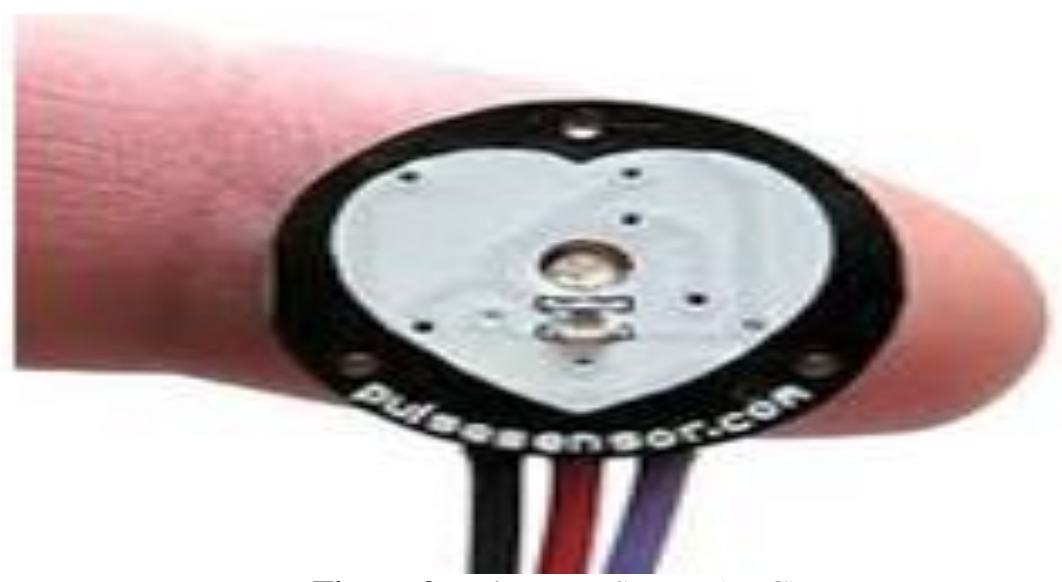

Figure 3. Pulse Rate Sensor (PPG)

E) GPS Module: GPS module in our circuit shown in Figure 4 is used to get the latitudes and longitude values of the user at the time of emergency. These latitudes and longitude values are sent to the emergency contacts with the help of the GSM module.

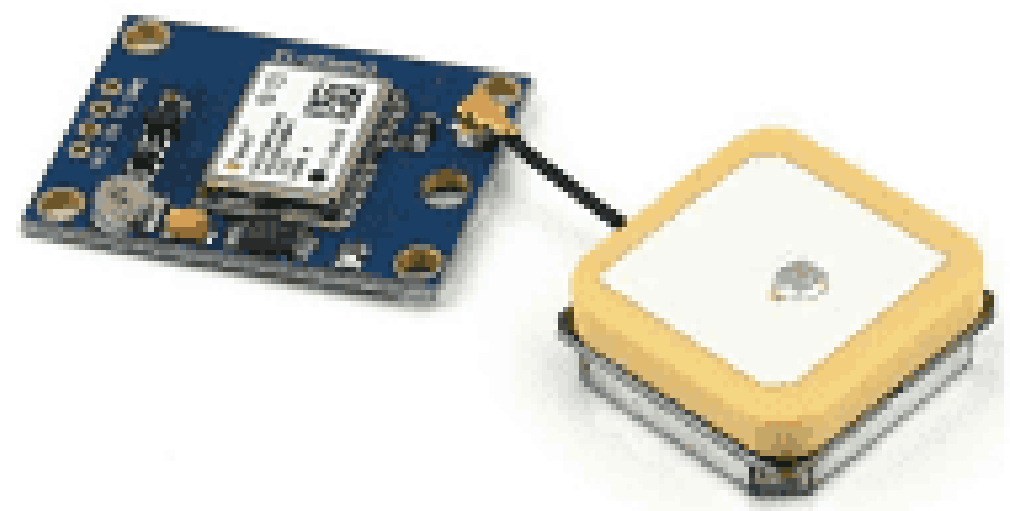

Figure 4. GPS Module 
F) GSM Module: The GPS coordinates along with alert messages are sent through GSM module to the emergency contacts at the time of emergency. This works directly with the help of mobile network through a sim card implanted in the module. The module is shown in Figure 5.

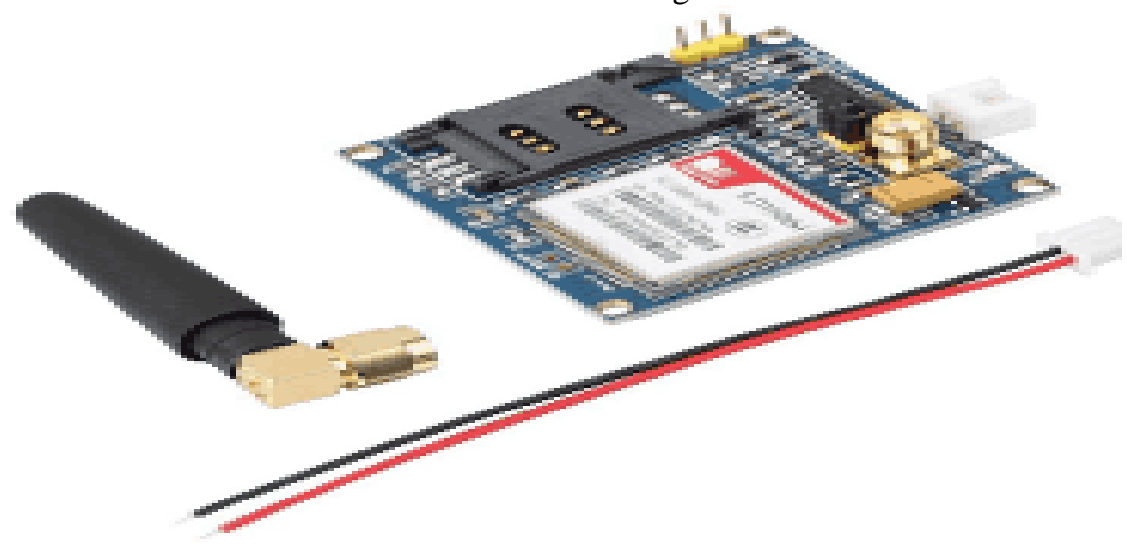

Figure 5. GSM Module

G) MicroVibration Motor: The vibration motor shown in Figure 6 is placed in the setup in order to ask the user whether he/she is safe or not that is for notifying the user. If he/she is safe then the user responds by pressing the mode button. This is used to minimize false triggers.

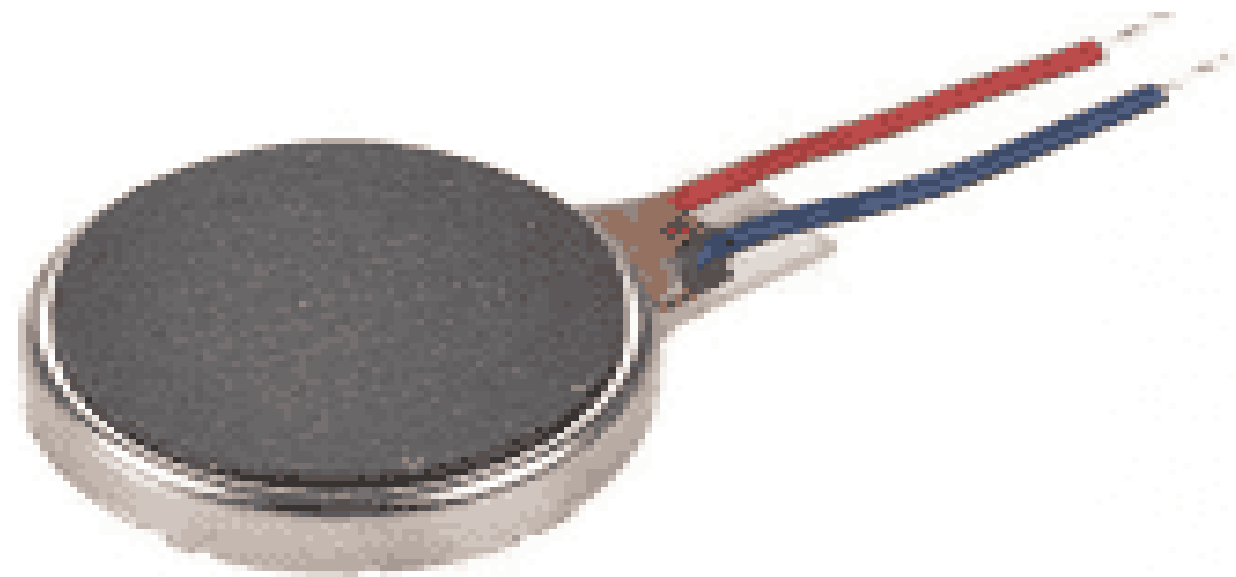

Figure 6. Vibration Motor

\subsection{Hardware Implementation}

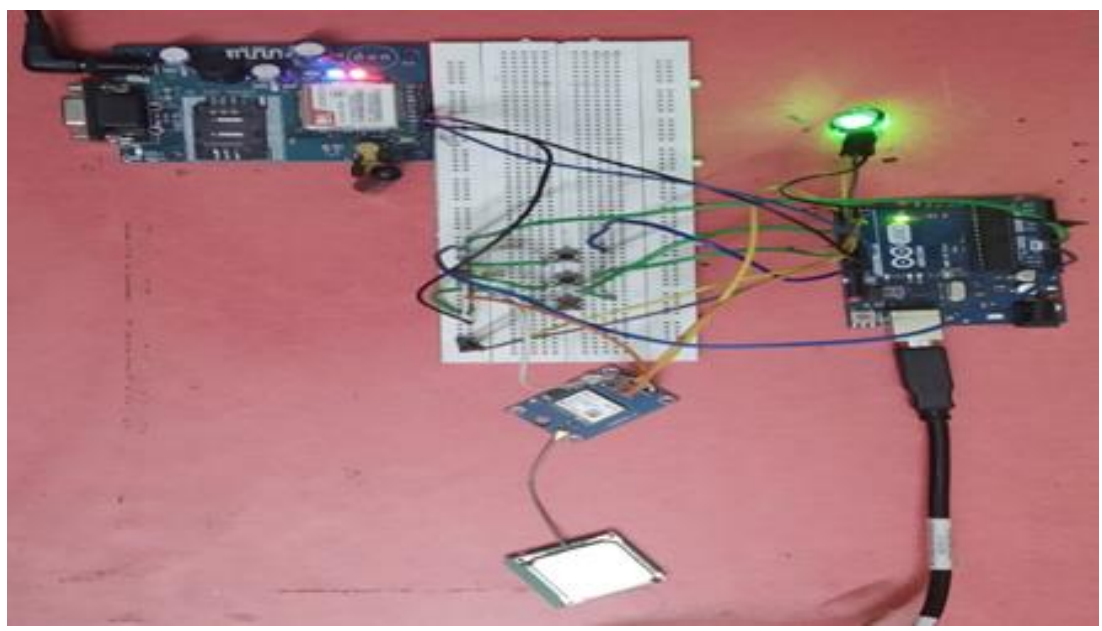

Figure 7. Hardware Implementation

Circuitin Figure 7 shows the implementation of our hardware all the modules used in the design like GPS, GSM and Arduino Uno and the three push buttons. The one in the red colour is the Emergency button, the one in 
the middle is the mode button whose functionality is explained above and the third button is for sleep. And LED at pin no: 13 in the arduinoplay the role of the vibration sensor.

\section{Result}

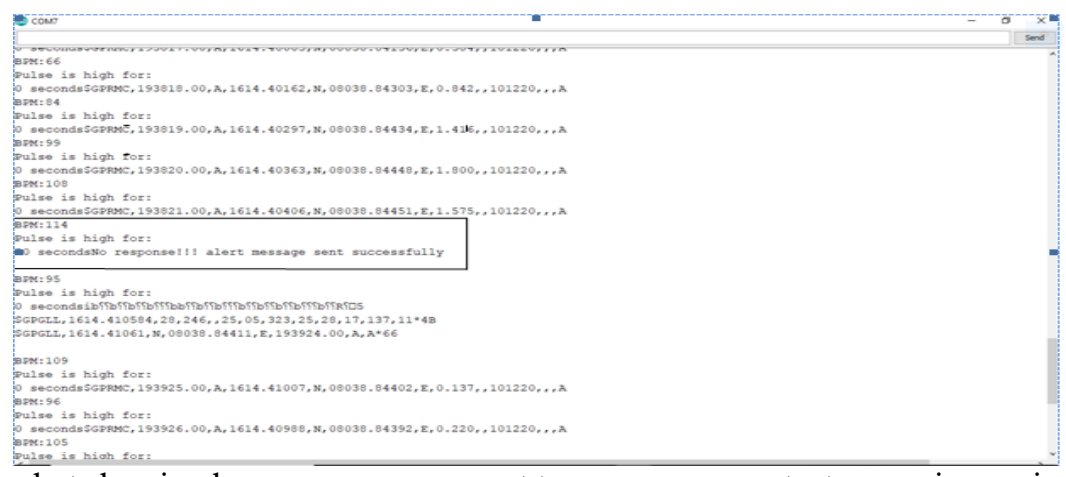

Figure 8. Snapshot showing how messages are sent to emergency contacts upon increasing the heart rate

Snapshot in Figure 8 shows that, as we have set an abnormal heart value of 110 in our design. The heart rate there has gone beyond 110 . So, it checked whether it would be greater than that particular abnormal heart rate value for at least 30 seconds or not. After that it sends us the verification signal through vibration sensor once in every 2 seconds for the next 30 seconds. In the above case we did not respond for the vibration and we left the mode button unpressed. Then immediately the device generated the following alert message Figure 9 along with the Google maps location of that particular area.

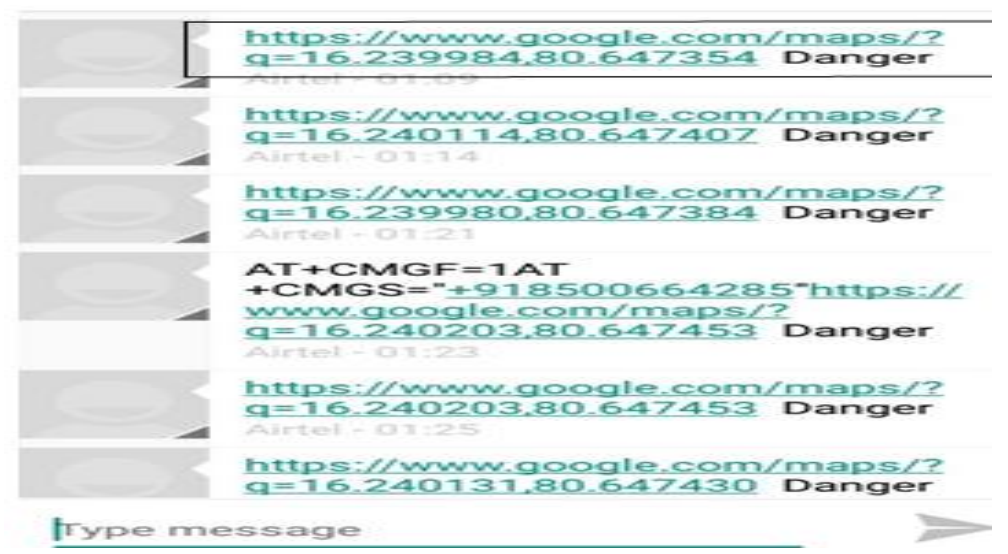

Figure 9. Snapshot of the alert message sent to emergency contact

The snapshotFigure 10 shows you how the emergency button works whenever required. Even, when the device is in sleep.

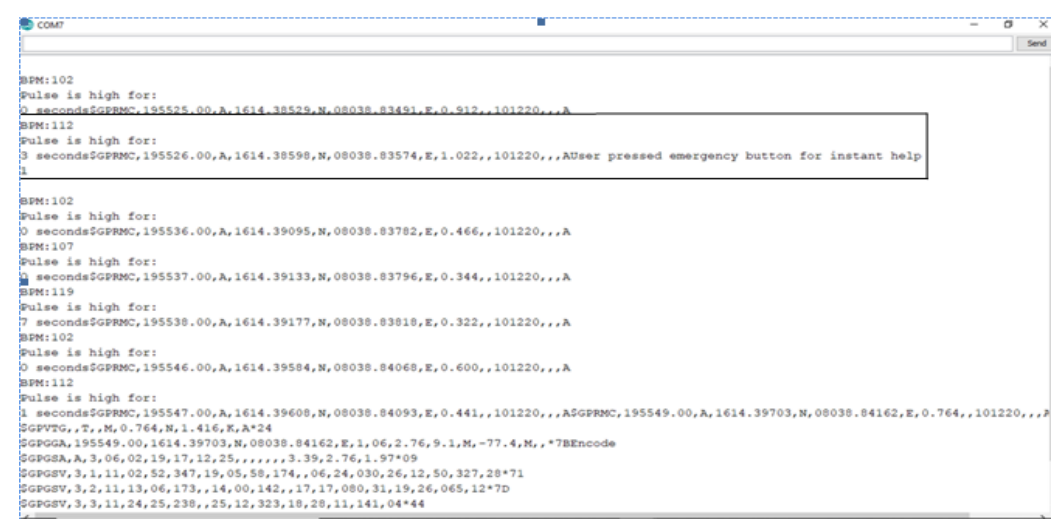

Figure10. Snapshot showing how the emergency messages are sent upon pressing the emergency button 
After pressing the emergency button it displayed the above message. Figure 10 and sent the following alert message shown in Figure 11 without any delay.

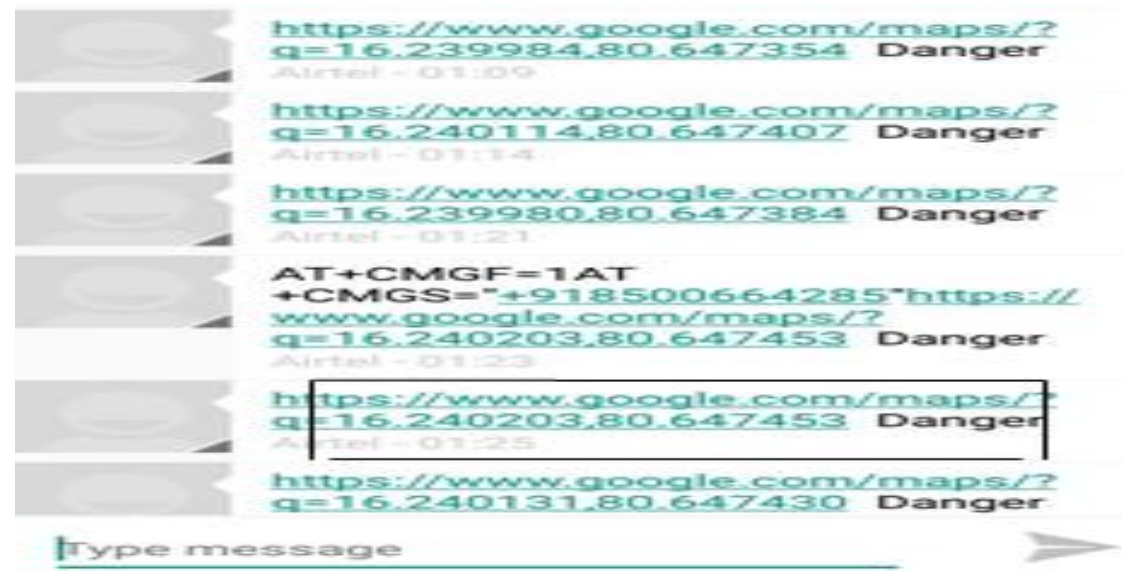

Figure 11. Alert message sent to emergency contact upon pressing the emergency button

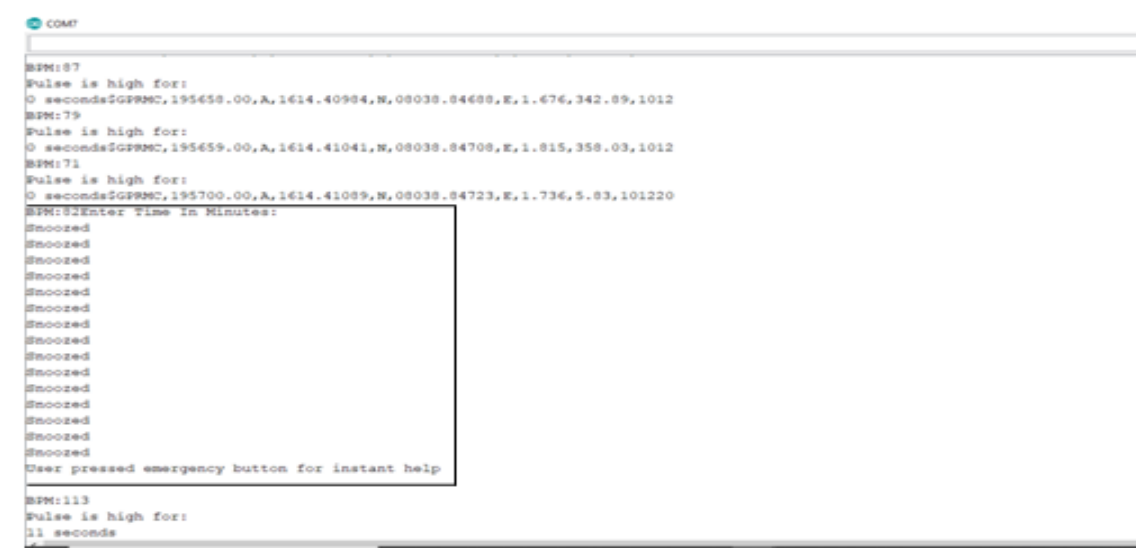

Figure 12.Snapshot showing how messages are sent even when the device is in sleep

The above snapshot in Figure 12 shows the working of the emergency button even in the sleep mode.

\section{Conclusion and Future Scope}

Using this model we can reduce the false triggers which may sometimes be fatal. As we designed the idea in the form of a prototype we did not include those ideas which are impossible to implement in the prototyping level. We are trying to develop this idea into a product especially in the form of a wearable using the nanotechnology and embedded systems. We are trying to develop the device in such a way that it should work in each and every situation possible. We will try to develop the fully loaded version of this device with all the ideas which we were unable to include in this design.

\section{References}

1. IoT Based Women Safety Device using ARM7 Shubham Sharma1, Fasil Ayaz2, Rajan Sharma3 , Divya Jain4 BE Student1, 2, 3 , Assistant Professor4 Department of ECE MIET, Jammu, India.

2. SMARISA: A Raspberry Pi based Smart Ring for Women Safety Using IoTNavya R Sogi, PriyaChatterjee, Nethra U, SumaV.

3. R, T. M., Aishwarya, S, C. K., K, D. M., \& H, N. (2019). IoT Based Smart Security Gadget for Women's Safety.

4. Sharma, V., Tomar, Y., \&Vydeki, D. (2019). Smart Shoe for Women Safety. 2019 IEEE 10th International Conference on Awareness Science and Technology (iCAST).

5. Ruman, M. R., Badhon, J. K., \&Saha, S. (2019). Safety Assistant and Harassment Prevention For Women. 2019 5th International Conference on Advances in Electrical Engineering (ICAEE).

6. Chand, D., Nayak, S., Bhat, K. S., Parikh, S., Singh, Y., \&Kamath, A. A. (2015). A mobile application for Women's Safety: WoSApp. TENCON 2015 - 2015 IEEE Region 10 Conference. 
7. Helen, A., Fathila, M. F., Rijwana, R., \&Kalaiselvi V.K.G. (2017). A smart watch for women security based on IoT concept "watch me.

8. Yarrabothu, R. S., \&Thota, B. (2015). Abhaya: An Android App for the safety of women. 2015 Annual IEEE India Conference (INDICON). doi:10.1109/indicon.2015.7443652.

9. Dr. SeetaiahKilaru, Hari Kishore K, Sravani T, AnveshChowdary L, Balaji T "Review and Analysis of Promising Technologies with Respect to fifth Generation Networks", 2014 First International Conference on Networks \& Soft Computing, ISSN:978-1-4799-3486-7/14,pp.270-273,August2014.

10. A Murali, K Hari Kishore, C P Rama Krishna, S Kumar, A TrinadhaRao "Integrating the Reconfigurable Devices using Slow-changing Key Technique to achieve High Performance "Proceedings- 7th IEEE International Advance Computing Conference, IACC 2017, 7976849 ISSN: 2473-3571, pp.530-534, July 2017.

11. AvinashYadlapati, KakarlaHari Kishore, "Constrained Level Validation of Serial Peripheral Interface Protocol", Proceedings of the First International Conference on SCI 2016, Volume 1, Smart Innovation, Systems and Technologies 77 (Publisher: Springer Nature Singapore Pvt. Ltd), ISSN No: 2190-3018, ISBN: 978-981-10-5544-7, Chapter No: 77, pp. 743-753, 25th December 2017.

12. Mahesh Madavath, Hari Kishore Kakarla, Azham Hussain, C.S. Boopathi“"Design and Analysis of CMOS RF Receiver Front-End of LNA for Wireless Applications” Microprocessors and Microsystems, ISSN: 0141-9331, Volume-75, Article: 102999, June 2020

13. AvinashYadlapati, Hari Kishore Kakarla“Low-power design-for-test implementation on phase-locked loop design" Measurement and Control, ISSN: 0020-2940, Volume-52, Issue No: (7-8), Page No: 995 1001, June 2019.

14. Nan Jiang, AbdolGhaffarEbadi, KakarlaHari Kishore, Qahtan. A. Yousif, Mohammad Salmani "Thermomechanical Reliability Assessment of Solder Joints in a Photo-voltaic Module Operated in a Hot Climate" IEEE Transactions on Components, Packaging and Manufacturing Technology, P-ISSN: 2156-3950, E-ISSN: 2156-3985, Vol No: 10, Issue No: 1, Page No: 160-167, January 2020.

15. M. Kavitha, Zaid Hamid Mahmoud, KakarlaHari Kishore, A.M. Petrov, AleksandrLekomtsev, Pavellliushin, Angelina OlegovnaZekiy, Mohammad Salmani “Application of Steinberg Model for Vibration Lifetime Evaluation of $\mathrm{Sn}-\mathrm{Ag}-\mathrm{Cu}$ based Solder Joints in Power Semiconductor" IEEE Transactions on Components, Packaging and Manufacturing Technology, P-ISSN: 2156-3950, E-ISSN: 2156-3985, January 2021.

16. Surendar, K. H. Kishore, M. Kavitha, A. Z. Ibatova, V. Samavatian "Effects of Thermo-Mechanical Fatigue and Low Cycle Fatigue Interaction on Performance of Solder Joints" IEEE Transactions on Device and Materials Reliability, P-ISSN: 1530-4388, E-ISSN: 1558-2574, Vol No: 18, Issue No: 4, Page No: 606-612, December-2018.

17. A Murali, K Hari Kishore, G A AnithaPriyadarshini "Improved design debugging architecture using low power serial communication protocols for signal processing applications" International Journal of Speech Technology (Springer), ISSN No: 1572-8110, January 2021.

18. Raja KumariChilukuri, Hari Kishore Kakarla, K SubbaRao "Estimation of Modulation Parameters of LPI Radar using Cyclostationary Design of Reconfigurable Low Power Pipelined ADC for BioImpedance Measurement" Sensing and Imaging, ISSN: 1557-2072, Volume-51, Issue-1, October 2020.

19. B Srikanth, M Siva Kumar, J V R Ravindra, K Hari Kishore "The enhancement of security measures in advanced encryption standard using double precision floating point multiplication model" Transactions on Emerging Telecommunications Technologies, ISSN: 2161-3915, Volume: 31, Issue: 0, June 2020.

20. K Hari Kishore, FazalNoorbasha, KattaSandeep, D. N. V. Bhupesh, SK. Khadar Imran, K. Sowmya "Linear convolution using UT Vedic multiplier" International Journal of Engineering and Technology(UAE), ISSN No: 2227-524X, Vol No: 7, Issue No: 2.8, Page No: 409-418, March 2018.

21. Nadhindla Bala Dastagiri, KakarlaHari Kishore, Vinit Kumar Gunjan and ShaikFahimuddin, "Design of a Low-Power Low-Kickback-Noise Latched Dynamic Comparator for Cardiac Implantable Medical Device Applications", Proceedings of Lecture Notes in Electrical Engineering 434, pp. 637-645, ISSN No: 1876-1100, E-ISSN: 1876-1119, January 2018.

22. Mahesh Madavath, K Hari Kishore "RF Front-End Design of Inductorless CMOS LNA Circuit with Noise Cancellation Method for IoT Applications" International Journal of Innovative Technology and Exploring Engineering, ISSN: 2278-3075, Volume-8, Issue No: 6, Page No: 176-183, April 2019.

23. K. Sarath Chandra, K Hari Kishore "Electrical Characteristics of Double Gate FINFET under Different Modes of Operation" International Journal of Innovative Technology and Exploring Engineering, ISSN: 2278-3075, Volume-8, Issue No: 6, Page No: 172-175, April 2019.

24. P.Ramakrishna, M. Nagarani, K Hari Kishore "A Low Power 8-Bit Current-Steering DAC Using CMOS Technology" International Journal of Innovative Technology and Exploring Engineering, ISSN: 2278-3075, Volume-8, Issue No: 6, Page No: 137-140, April 2019. 
25. K Hari Kishore, FazalNoorbasha, KattaSandeep, D. N. V. Bhupesh, SK. Khadar Imran, K. Sowmya "Linear convolution using UT Vedic multiplier" International Journal of Engineering and Technology(UAE), ISSN No: 2227-524X, Vol No: 7, Issue No: 2.8, Page No: 409-418, March 2018.

26. K Hari Kishore, B. K. V. Prasad, Y. Manoj SaiTeja, D. Akhila, K. Nikhil Sai, P. Sravan Kumar "Design and comparative analysis of inexact speculative adder and multiplier" International Journal of Engineering and Technology(UAE), ISSN No: 2227-524X, Vol No: 7, Issue No: 2.8, Page No: 413426, March 2018.

27. Nadhindla Bala Dastagiri, KakarlaHari Kishore, Vinit Kumar Gunjan and ShaikFahimuddin, "Design of a Low-Power Low-Kickback-Noise Latched Dynamic Comparator for Cardiac Implantable Medical Device Applications", Proceedings of Lecture Notes in Electrical Engineering 434, pp. 637-645, ISSN No: 1876-1100, E-ISSN: 1876-1119, January 2018.

28. P.Ramakrishna, M. Nagarani, K Hari Kishore "A Low Power 8-Bit Current-Steering DAC Using CMOS Technology" International Journal of Innovative Technology and Exploring Engineering, ISSN: 2278-3075, Volume-8, Issue No: 6, Page No: 137-140, April 2019.

29. Mahesh Madavath, K Hari Kishore "RF Front-End Design of Inductorless CMOS LNA Circuit with Noise Cancellation Method for IoT Applications" International Journal of Innovative Technology and Exploring Engineering, ISSN: 2278-3075, Volume-8, Issue No: 6, Page No: 176-183, April 2019.

30. K. Sarath Chandra, K Hari Kishore "Electrical Characteristics of Double Gate FINFET under Different Modes of Operation" International Journal of Innovative Technology and Exploring Engineering, ISSN: 2278-3075, Volume-8, Issue No: 6, Page No: 172-175, April 2019.

31. AvinashYadlapati, K Hari Kishore "Implementation of Asynchronous FIFO using Low Power DFT" International Journal of Innovative Technology and Exploring Engineering, ISSN: 2278-3075, Volume8, Issue No: 6S, Page No: 152-156, April 2019.

32. ChellaSanthosh, K. Hari Kishore, G. Pavani Lakshmi, G. Kushwanth, P. Rama Krishna Dharma Teja, R. S. Ernest Ravindran, SreeVardhanCheerala, M. Ravi Kumar "Detection of Heavy Metal Ions using Star-Shaped Microfluidic Channel" International Journal of Emerging Trends in Engineering Research, ISSN: 2347-3983, Volume-7 Issue-12, Page No: 768-771, December 2019.

33. Mahesh Madavath, K Hari Kishore "Design and Analysis of Receiver Front-End of CMOS Cascode Common Source Stage with Inductive Degeneration Low Noise Amplifier on $65 \mathrm{~nm}$ Technology Process" Journal of Nanoscience and Nanotechnology, ISSN: 1546-1955, Volume-16, Issue No: (5-6), Page No: 2628-2634, June 2019.

34. P Ramakrishna, K Hari Kishore "Implementation of Low Power and Area Efficient 7-Bit Flash Analog to Digital Converter" Journal of Nanoscience and Nanotechnology, ISSN: 1546-1955, Volume-16, Issue No: (5-6), Page No: 2213-2217, June 2019.

35. Ch. Naga Babu, P. Naga Siva Sai, Ch. Priyanka, K Hari Kishore, M. BinduBhargavi, K. Karthik "Comparative Analysis of High Speed Carry Skip Adders" International Journal of Engineering and Technology (UAE), ISSN No: 2227-524X, Vol No: 7, Issue No: 2.24, Page No: 121-125, April 2018.

36. G SiriVennela, K Hari Kishore, E Raghuveera "High Accurate and Power Efficient ECG-Based Processor for Predicting Ventricular Arrhythmia" Journal of Advanced Research in Dynamical and Control Systems, ISSN No: 1943-023X, Vol No: 10, Issue No: 2, Page No: 1180-1121, May 2018.

37. P. Gopi Krishna, K. Sreenivasa Ravi, K Hari Kishore, K Krishna Veni, K. N. Siva Rao, R.D Prasad "Design and Development of Bi-Directional IoT gateway using ZigBee and Wi-Fi technologies with MQTT Protocol" International Journal of Engineering and Technology(UAE), ISSN No: 2227-524X, Vol No: 7, Issue No: 2.8, Page No: 125-129, March 2018.

38. Mahesh Madavath, K Hari Kishore "RF Front-End Design of Inductorless CMOS LNA Circuit with Noise Cancellation Method for IoT Applications" International Journal of Innovative Technology and Exploring Engineering, ISSN: 2278-3075, Volume-8, Issue No: 6, Page No: 176-183, April 2019

39. K DivyaMadhuri, K Hari Kishore "Implementation of 4-bit Ripple Carry Adder by Adopting Sub threshold Adiabatic Logic for Ultralow-Power Application" Journal of Advanced Research in Dynamical and Control Systems, ISSN No: 1943-023X, Vol No: 12, Issue No: 6, Page No: 11-17, May 2020.

40. Bhogadi Anil Kumar, ChillapalliHaritha, Gumpena Veda Sri Leela, E Raghuveera, K Hari Kishore“ A Parametric DFT Scheme for RAMs" Journal of Advanced Research in Dynamical and Control Systems, ISSN No: 1943-023X, Vol No: 12, Issue No: 2, Page No: 2298-2305, May 2020. 